\title{
Ink-Bottle Pore Changes in Cement Concrete Corroded by Environmental Coupling Effect
}

\author{
Lei $\mathrm{YU}^{1, \mathrm{a},{ }^{*}}$, Guo-Qing ZHANG ${ }^{2, \mathrm{~b}}$ and Zheng $\mathrm{LI}^{2, \mathrm{c}}$ \\ ${ }^{1}$ Research institute of highway ministry of transport, China \\ ${ }^{2}$ Beijing Xin-Qiao Technology Development Co., LTD, China \\ a270263604@qq.com, b875272455@qq.com, c 603718619@qq.com
}

Keywords: Cement Concrete, Environmental Factor, Coupling Effect, Ink-Bottle Pore.

\begin{abstract}
. 3 kinds of cement concretes are listed as follow: normal concrete, air-entrained concrete with $4 \%$ air content, concrete with $15 \%$ fly ash and $4 \%$ air content. Experiment methods of environmental coupling effect were designed. Model had been proposed in order to classify ink-bottle pore. Contents and shapes of ink-bottle pores had been compared among all kinds of ink-bottle pores in treatment group, control group samples. Result shows that ink-bottle pore content and shape are different between concretes corroded by environmental coupling effects and ones corroded by single environmental factor. Ball shape ink-bottle pore with 3 50nm pore size diameter makes an important role for stability of concrete. Ball shape ink-bottle pore is stronger than ellipsoid one. The more flat ellipsoid shape ink-bottle pore is, the weaker it is.
\end{abstract}

\section{Introduction}

Cement concrete is a kind of materials with a lot of little pores [1]. Pore shape changes will lead to pore structure changing [2]. Pore structure changing will lead to concrete macro performance change. Some particles with corrosion property delayed in cement pore will lead to pore expanded and destroyed and then lead to concrete destroyed [3].

In order to find pores degradation mechanism in concrete, different environments are simulated and pore shape changing rules under simulated environments are studied.

\section{Environmental Factors}

Dry-wet cycles (DW), chlorine salt erosion (Cl-), carbonization (C) and freeze-thaw cycles (FT) are simulated.

\section{Test Method}

Dry-wet cycles and chlorine salt erosion are simulated with Instruction Manual Salt Water Immersion and Drying Tester. Set dry temperature is $(50 \pm 5){ }^{\circ} \mathrm{C}$, dry time of duration is $24 \mathrm{~h}$. Salt water concentration is $(3 \pm 0.5) \%$. Salt water temperature is $(25 \pm 5){ }^{\circ} \mathrm{C}$ and $24 \mathrm{~h}$ corrosion. Dry-wet cycles 15 times (30d).

Specimen after Dry-wet cycles and salt erosion are put into carbonation tank. Set $\mathrm{CO}_{2}$

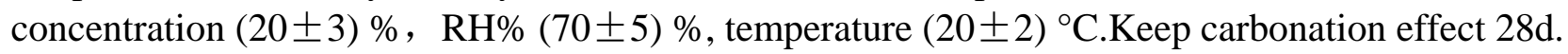

Specimen after dry-wet cycles and salt erosion and carbonation are put into freeze-thaw cycles machine. Set center lowest and highest temperature is $(-18 \pm 2)^{\circ}$ Cand $(5 \pm 2){ }^{\circ} \mathrm{C}$.

Use Mercury Injection Apparatus to test specimen pore parameters and curves [4]. 


\section{Specimen Prepared}

Raw Materials. P.O 42.5. Fly ash. Nature sand with 2.78 fineness modulus. Diameter range of coarse aggregate is $5-15 \mathrm{~mm}$. Tap water. Naphthalene water reducer with $20 \%$ water-reducing rate. Colophony air-entraining agent ( $0.01 \%$ mix content corresponding 3\% air content).

Mix Proportion. 3 kinds of cement concrete has been made: 1\# normal cement concrete, 2\# 4\% air content cement concrete, $3 \# 4 \%$ air content and $15 \%$ fly ash mixed cement concrete. Mix proportions of 3 kinds of concretes are shown as table 1.

Table 1. Mix Proportion of 3 kinds of concretes

\begin{tabular}{cccccc}
\hline ID & cement & fly ash & water & sand & $\begin{array}{c}\text { aggregat } \\
\text { e }\end{array}$ \\
\hline $1 \#$ & 542 & 0 & 220 & 573 & 1065 \\
$2 \#$ & 542 & 0 & 220 & 573 & 1065 \\
3\# & 461 & 81 & 220 & 573 & 1065 \\
\hline
\end{tabular}

Note: Keep fluidity of 3 kinds of concretes with $(10 \pm 3) \mathrm{cm}$.

Number Specimens. According to different environment effects, specimens are numbered as shown as table 2 .

Table 2. Test Group, Identifier and Environmental Factors Corroded

\begin{tabular}{lllllll}
\hline \multirow{2}{*}{ Environment factors } & Group & ID & $\begin{array}{l}\text { Curing } \\
(28 d)\end{array}$ & $\begin{array}{l}\text { DW+Cl } \\
\text { (30d) }\end{array}$ & $\begin{array}{l}\text { C } \\
\text { (28d }\end{array}$ & FT \\
\hline 28d Curing & Curing group & CG1 & $\triangle$ & $X$ & $X$ & $X$ \\
DW $+\mathrm{Cl}^{-}$ & Control group & DW-CG & $\triangle$ & $\triangle$ & $X$ & $X$ \\
& Test group & DW-TG & $\triangle$ & $\sqrt{ }$ & $X$ & $X$ \\
C & Curing group & CG2 & $\triangle$ & $\triangle$ & $\triangle$ & $X$ \\
& Control group A & C-CG & $\triangle$ & $\sqrt{ }$ & $\triangle$ & $X$ \\
& Test group & C-TG & $\triangle$ & $\sqrt{ }$ & $\sqrt{ }$ & $X$ \\
FT & Curing group & CG3 & $\triangle$ & $\triangle$ & $\triangle$ & $\triangle$ \\
& Control group B & FT-CG & $\triangle$ & $\sqrt{ }$ & $\sqrt{ }$ & $\triangle$ \\
& Test group & FT-TG & $\triangle$ & $\sqrt{ }$ & $\sqrt{ }$ & $\sqrt{ }$ \\
\hline
\end{tabular}

Note: $\triangle$ - curing; $\sqrt{ }$ - corrosion; $X$ - no corrosion.

\section{Ink-Bottle Pore Model}

Ink-bottle pore has small neck and large tummy like pockets. This kind of pore structure is good for adsorption capacity and leading to mass transfer rate [5].

Ink-bottle pore shape can be described by two parameters such as pore neck diameter and pore cavity volume. Ink-bottle pore shapes are all different. While, it can be separated into two kinds. One is ball shape ink-bottle pore (BP), the other is ellipsoid shape ink-bottle pore (EP). Define pore neck diameter as $d_{n}$. Define pore cavity diameter as $d_{e}$. So pore cavity volume can be calculated as $V_{e}=\frac{1}{6} \pi d_{e}^{3}$,

$$
\text { If } d_{n}=d_{e}, \frac{d_{n}}{V_{e}}=\frac{6}{\pi d_{n}^{2}} \text {; }
$$


If $d_{n}<d_{e}$ 时, $\frac{d_{n}}{V_{e}}<\frac{6}{\pi d_{n}^{2}}$;

If $d_{n}>d_{e}$ 时, $\frac{d_{n}}{V_{e}}>\frac{6}{\pi d_{n}^{2}}$.

Define $\frac{d_{n}}{V_{e}}$ as $\Omega$. It is supposed to an index to describe ink-bottle pore shape. When $\Omega<\frac{6}{\pi d_{n}^{2}}$, pore neck diameter is smaller than pore cavity diameter, $d_{n}<d_{e}$, pore model can be described as figure 1 . When $\Omega>\frac{6}{\pi d_{n}^{2}}$, pore neck diameter is larger than pore cavity diameter, $d_{n}>d_{e}$, pore model can be described as figure 2 .
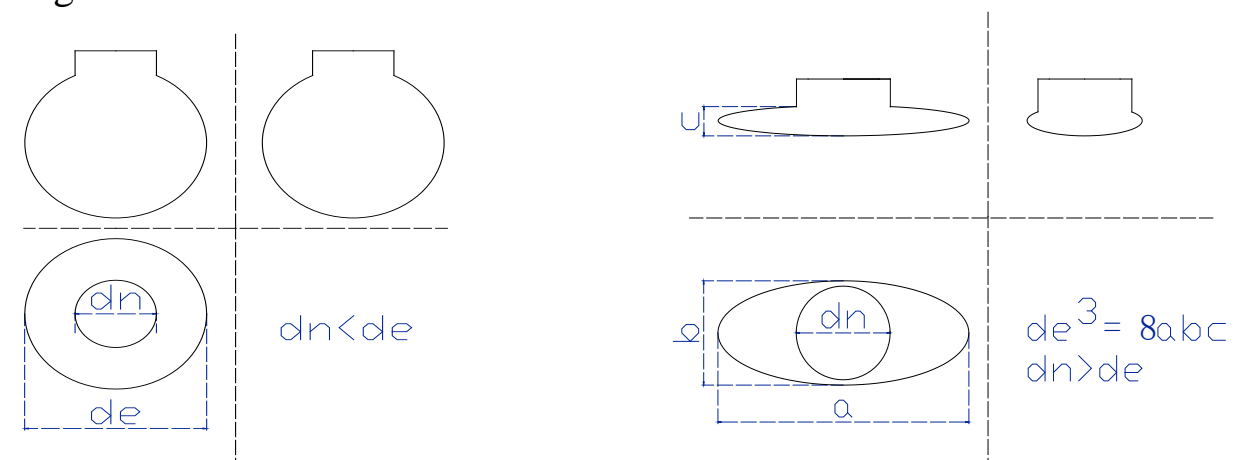

Fig.1 Model for Ball Shape Ink-bottle Pore Fig.2 Model for Ellipsoid Shape Ink-bottle Pore

Difference mercury injection and ejection volume can be calculated according to mercury injection and ejection accumulative curve. Stranded mercury volume are similar to ink-bottle pore volume $V_{e}$. $d_{e}$ can be calculated by volume of ink-bottle pore. Calculated $\Omega$ when $d_{n}=d_{e}$ and compared to real $\Omega$. According to comparing result and figure 1 and 2, separate ink-bottle pore style.

\section{Test Result Analysis}

Figure 4 to figure 6 are shape variations of ink-bottle pore in cement concrete with different environment effected.

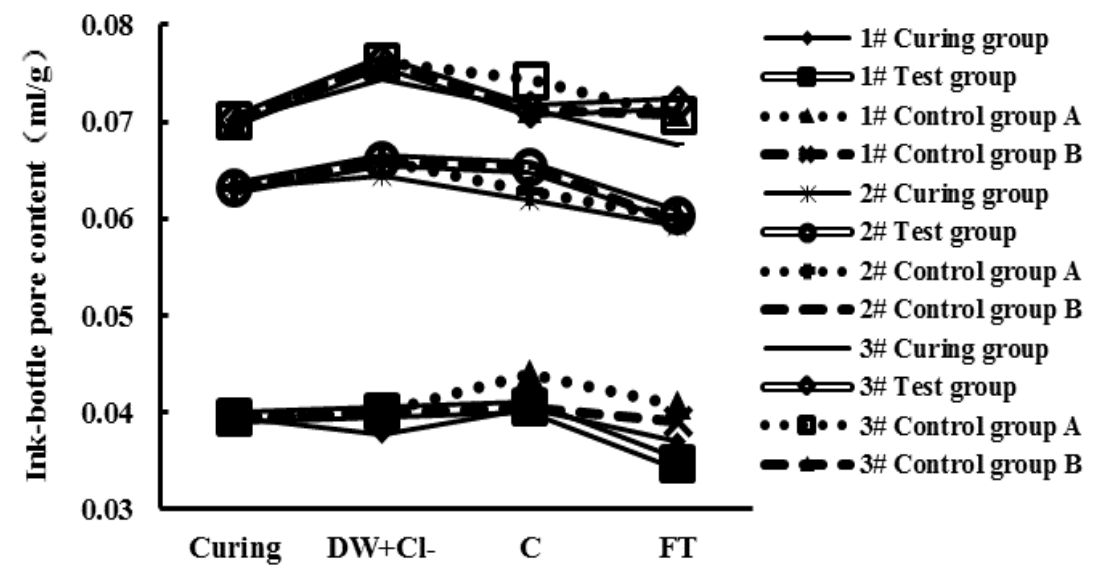

Fig.3 Ink-bottle Pore Content of Concrete by Environmental Coupling Effect

Figure 3 shows that ink-bottle pore content is effected by mix proportion remarkably. $1 \#<2 \#<3 \#$. 3 kinds of concrete ink-bottle pore content increased a little after dry and wet recycle and chlorine salt 
erosion coupling effected. After dry and wet recycle and chlorine salt erosion and carbonization, 2\# concrete ink-bottle pore content increased a little. 1\# and 3\# keep steady. After dry and wet recycle and chlorine salt erosion and carbonization and frozen and thaw recycle, content of ink-bottle pore in 1\# concrete decreases remarkably. Ink-bottle pore Content of 3\# concrete increases. 2\# keep steady.

Comparing control group A and test group, after single carbonization erosion, ink-bottle pore content of 2\# concrete increases.

Comparing control group B and test group, after single frozen and thaw recycle erosion, ink-bottle pore content of 2\# concrete keep steady.

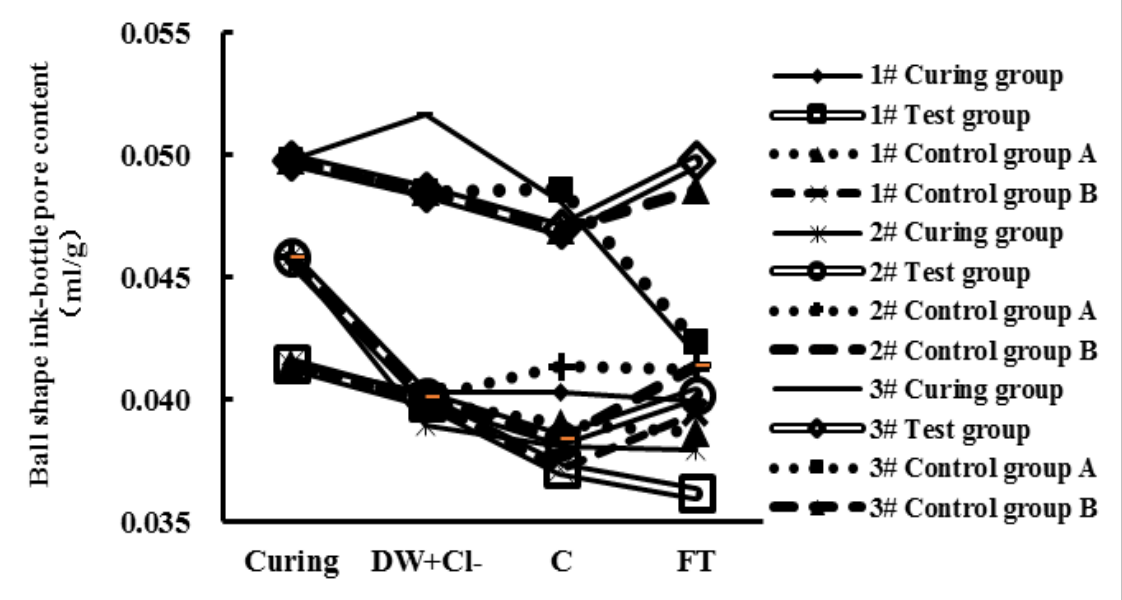

Fig.4 Ball shape ink-bottle Pore Content of Concrete Corroded by Environmental Coupling Effects

From figure 4, ball shape ink-bottle pore diameters mainly distributes among $2 \mathrm{~nm}$ to $50 \mathrm{~nm}$. Ellipsoid shape ink-bottle pore diameter mainly distributes among 50 to 2000nm. Figure 5 shows that after 4 environment factors coupling effects, content of ball shape ink-bottle pore in $3 \#$ concrete is the most. The second is $2 \#$ concrete. The least is $1 \#$ concrete.

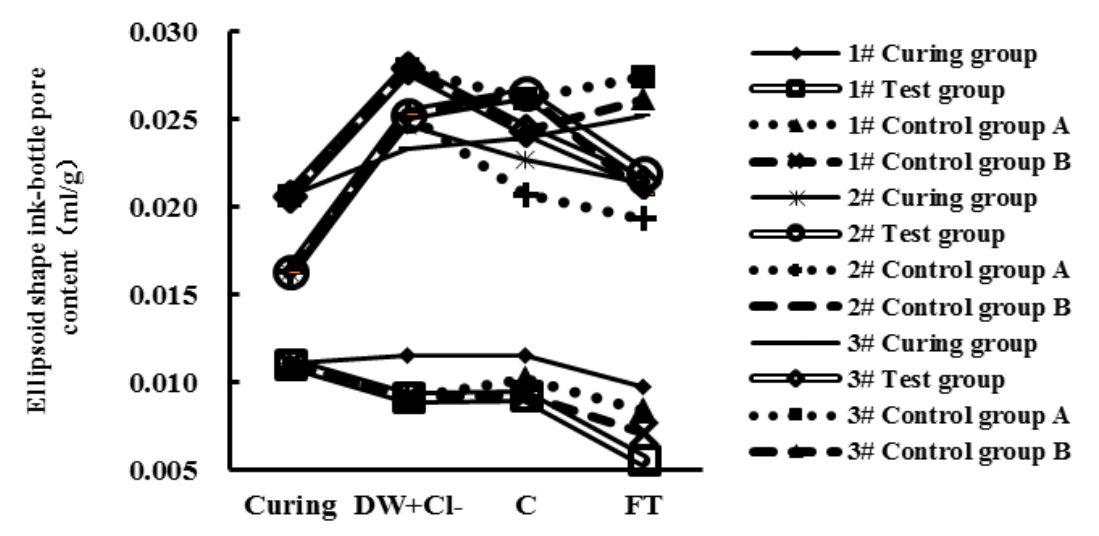

Fig.5 Ellipsoidal Shape ink-bottle Pore Content of Concrete Corroded by Environmental Coupling Effects

Figure 5 shows that after 4 environment factors, content of ellipsoid shape ink-bottle pore in $3 \#$ concrete is the most. Second is 2\# concrete. The least is 1\# concrete. Content of ellipsoid shape ink-bottle pore in $1 \#$ concrete decreased one by one environment factor erosion. After carbonization coupling, ellipsoid shape ink-bottle pore content of 2\# concrete increases most among 3 concretes. After freeze-thaw cycle coupling, ellipsoid shape ink-bottle pore content of 3\# concrete decreases most among 3 kinds of concretes. 


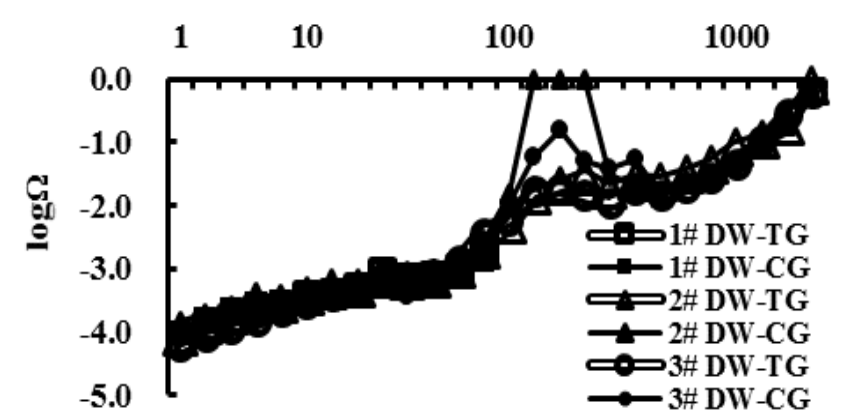

Fig. $6 \Omega$ of Ink-Bottle Pore in Concrete Corroded by Dry-wet Cycle and Chloride Erosion

Figure 6 shows that $\Omega$ has no significant variation in $1 \#$ concrete after dry and wet recycle and chloride erosion. Ink-bottle pore distribute among 3-2000nm in $2 \#$ and $3 \#$ concrete. Ball shape ink-bottle pore diameters distribute among $3-50 \mathrm{~nm}$. $\Omega$ of ball pore has no significant variation while $\Omega$ of ellipsoid pore decreases significantly.

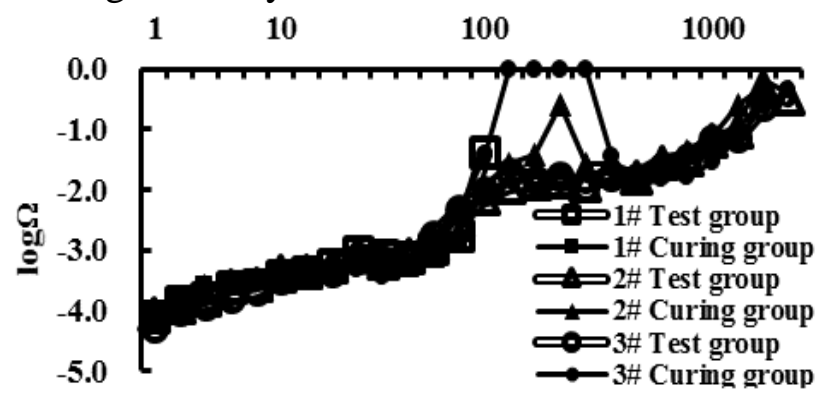

Fig.7 $\Omega$ of Ink-bottle Pore in Concrete Corroded by Dry-wet Cycle, Chloride Erosion and Carbonization

Figure 7 shows that $1 \#$ concrete ink-bottle pore has no significance change after 3 environment factors coupling. Ball shape ink-bottle pore has no significance change in $2 \#$ and $3 \#$ concrete. $\Omega$ of ellipsoid shape ink-bottle pore decreases.

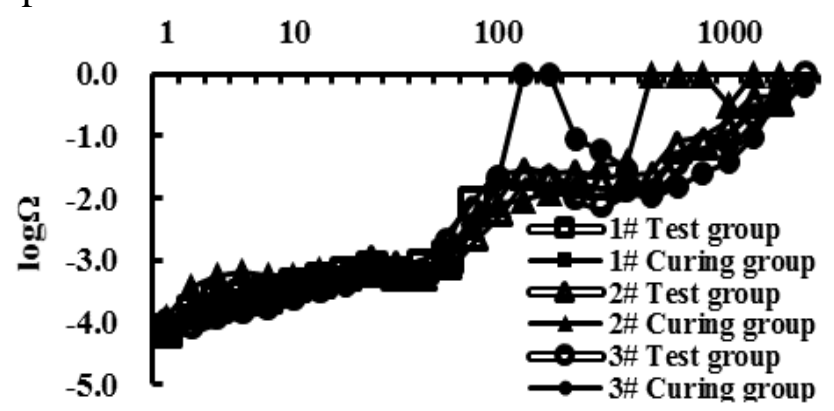

Fig. $8 \Omega$ of Ink-bottle Pore in Concrete Corroded by Dry-wet Cycle, Chloride Erosion, Carbonization and Freeze-thaw Cycle

As shown as figure 8 , after 4 environment factors coupling effects, shape of $1 \#$ ink-bottle pore has no significant change. $\Omega$ of 3-10nm ball shape ink-bottle pore in $2 \#$ concrete decreases. $200-2000 \mathrm{~nm}$ ellipsoid shape ink-bottle pore in $2 \#$ concrete decreases. $\Omega$ of $1-10 \mathrm{~nm}$ ball shape ink-bottle pore in 3\# concrete decreases. 100-500nm ellipsoid shape ink-bottle pore in 3\# concrete decreases. 500-2000nm ellipsoid shape ink-bottle pore in $3 \#$ concrete increases a little. So, in different mix proportion concretes, variation of ball shape ink-bottle pores contributes more than ellipsoid shape ones for pore structure steady.

\section{Summary}

3 kinds of concrete are made to study variations of ink-bottle pore shapes after 4 environment factors coupling. Conclusions are as follow: 
Define $\Omega$ as a parameter to describe ink-bottle pore shape. 2 models of ink-bottle pore are put out. One is ball shape ink-bottle pore model and the other is ellipsoid shape ink-bottle pore model. Ball shape ink-bottle pore is stronger than ellipsoid ones in aspect of structure steady. The flatter ellipsoid shape ink-bottle pore is, the weaker it is.

After 4 environment factors coupling, ink-bottle pore shape has no significant variation for $1 \#$ concrete. While for 2\# and 3\# concrete, ink-bottle shape become homogenizing.

In different mix proportion concretes, variation of ball shape ink-bottle pores plays more important role than ellipsoid shape ones for pore structure steady.

\section{References}

[1] Jia-Ming ZHENG. Research on the sulfate attack of concrete under the dry and wet cycles and carbonation [D]. Yang Zhou University, 2009.

[2] Lei YU, Jun ZHANG, Jin-Xi ZHANG et.al. Quantitative Relation model between macro performance and pre structure of cement concrete [J]. 2015, 36(11), pp. 1459-1464.

[3] Jun-Hui HE. Study on Microstructure and Properties of Road Cement Concrete.2009:3-5.

[4] Hong-Mei WANG. Mercury Determination the Error of Structure of Porous Material [J]. Guangzhou Chemicals, 2009, 37 (1), pp.109-111.

[5] Ying ZHANG, Liang-Xu LIN, Zi-Feng YAN et.al. Study on Ink-Bottle Pore Preparation Using SBA-15 as Template Based on MOCVD New Method [J]. Chinese Science Bulletin, 2009, 54 (14), pp.2113-2117. 